\title{
KINETICS OF DENSIFICATION BY SOLUTION-REPRECIPITATION
}

\author{
A. MORTENSEN + \\ Department of Materials Science and Engineering, Massachusetts Institute of Technology, Cambridge, \\ Massachusetts. U.S.A.
}

(Received 19 April 1996)

\begin{abstract}
The densification rate and microstructural evolution of powder compacts densifying by solution-reprecipitation in the presence of a liquid phase are predicted with the assumption that solid particles are equisized spheres randomly packed and pulled together by a liquid that wets perfectly both the solid and its grain boundaries. The influences of applied pressure, initial packing density, and of the "sphering force" due to changes in solid particle shape are accounted for. The resulting sintering rate retains approximately the one-third power law dependence of linear shrinkage on time predicted by Kingery, and shows good agreement with published experimental data. The analysis is extended to include the role of particle coarsening. so as to assess its importance and influence in densification kinetics. Copyright 1997 Acta Metallurgica Inc.
\end{abstract}

\section{INTRODUCTION}

Liquid phase sintering is one of the most industrially significant powder densification processes. In its usual form, this process consists of heating, sometimes under pressure, a mixed powder compact until a small amount of chemically stable liquid phase forms and greatly accelerates sintering of the powders. Because of its practical importance, a large body of research, summarized in two monographs $[1,2]$, has been conducted on liquid phase sintering, towards identification and quantification of the rate of sintering, and towards prediction of the resulting material microstructure.

There is broad agreement that, despite considerable microstructural complexity and system-tosystem specificities, the densification of liquid phase sintered structures proceeds essentially in three stages. The first of these is a relatively rapid rearrangement of solid particles, which begins immediately upon formation of the liquid. The second stage, solution-reprecipitation, is observed only in systems featuring some solubility of the remaining solid phase in the liquid phase. In the third stage, microstructural coarsening is observed in many systems, accompanied by much slower solid-state sintering in systems where there is formation of a network of solid bridges between touching solid particles.

The first stage, rearrangement, is geometrically complex. When the liquid phase forms, the remaining solid phase particles change position under the action

$\lceil$ At the time of writing on sabbatical leave at: Centre des Matériaux Pierre-Marie Fourt, Ecole Nationale Supérieure des Mines de Paris, Corbeil, France. of capillary forces, to eliminate void spaces within the structure. Rearrangement is complicated by several capillary and dissolution phenomena; however, in the simpler systems, the observed densification kinetics are predominantly limited by viscous flow of the liquid. Hence, as first shown by Kingery [3], the rate of densification during rearrangement is roughly constant, and inversely proportional to the solid particle radius if monosized solid phase powders are used. Rearrangement stops either when the liquid fills all pore space present, or when the solid particles are packed to the maximum volumetric density that can be attained from the initial mixed powder structure without deformation of the solid particles.

When rearrangement does not fully densify the compact, solution-reprecipitation can provide a rapid alternate densification path in systems where there is some solubility of the solid in the liquid phase. Solution-reprecipitation is the process whereby solid phase material along contact areas between touching solid particles dissolves into the liquid, and deposits elsewhere along the liquid/solid interface. The net result of this process is that solid particles are deformed, in a manner that causes an increase in their packing density and, hence, a reduction in pore volume within the powder compact. Resulting microstructures feature, in non-faceted solid/liquid systems, flattened spheres typical of the "grain shape accommodation" that is observed, for example, in heavy alloy microstructures (examples are given on pages 7 and 104 of the monograph by German [2]).

Despite considerable research on the kinetics of densification during this second, solutionreprecipitation, phase of liquid phase sintering, few quantitative models exist. Essentially, the only quantitative prediction of the rate of densification 
during this stage of the process is that of Kingery $[4,5]$, which equates the linear rate of shrinkage of a powder compact with that of two isolated particles wetted at their contact region by the liquid. Kingery's analysis predicts that the relative shrinkage of a powder compact during sintering by solutionreprecipitation is proportional to $r^{-4 / 3} t^{1 / 3}$, where $r$ is the solid powder particle radius, and $t$ is time.

Despite clear substantiation of this prediction by experiment $[1,2,6,7]$, Kingery's model has been criticized on the basis that it is too simple. Indeed, it ignores the possible influence of several phenomena, including solid phase particle coarsening, which is necessarily present to some extent during sintering by solution-reprecipitation [2, 8, 9]. Significant criticism of Kingery's model can also be voiced on the same grounds as with similar early models of solid state sintering, namely that consideration of two touching particles only is an oversimplification of the microstructure of a densifying powder compact.

Significant advances have been accomplished over the past decade in the theoretical analysis of solid-state powder densification. The outcome of this research effort is a coherent microstructural framework for analysis of solid state densification, bascd on the random close packed sphere model. Resulting equations agree with experiment, and show that analyses of powder densification based on twoparticle models are, indeed, oversimplified [10-13].

This article provides a quantitative analysis of the kinetics of liquid phase sintering by solutionreprecipitation which relieves several of the assumptions inherent in Kingery's model, yet recovers the essential predictions of that model, its good agreement with experiment, and the overall physical insight it first provided. Beyond relieving several of Kingery's assumptions, the present model provides a basis for the analysis of various pertinent phenomena, such as the presence of large pores in the final microstructure, the influence of coarsening on densification rate, the formation of solid volume fraction gradients under the influence of gravity, and macroscopic liquid flow across densified liquid phase sintered structures.

We begin by presenting the assumptions we make, then derive governing equations of densification by liquid phase sintering, and then discuss their implications and extension to a coarsening system in the final main section of this article.

\section{PRINCIPAL ASSUMPTIONS}

We consider a compact densifying by solutionreprecipitation during liquid phase sintering, immediately after melting of the liquid and rearrangement of the powder particles under the action of capillary forces. We focus on the simplest geometrical case, namely one for which the following assumptions hold. (i) The solid/liquid interfacial energy is isotropic. Consequently we assume that there is no faceting of the solid particles.

(ii) The liquid wets the solid perfectly:

$$
\sigma_{\mathrm{sl}}+\sigma_{\mathrm{lv}} \leqslant \sigma_{\mathrm{sv}}
$$

where $\sigma$ denotes interfacial energy, and subscripts s, 1 and $v$ the solid, liquid, and vapour phases respectively. Pores are therefore separated from the solid/liquid boundary by at least a thin film of liquid. We do not expect slight violation of this criterion to introduce large deviations in predictions of the present analysis.

(iii) The liquid penetrates grain boundaries of the solid phase:

$$
2 \sigma_{\mathrm{sl}}<\sigma_{\mathrm{gb}}
$$

where $\sigma_{\mathrm{gb}}$ is the grain boundary energy of the solid phase. Therefore, solid grains are entirely surrounded by liquid, a thin film of which is always present between neighbouring grains of the solid phase.

(iv) The compact densifies by the diffusioncontrolled solution-reprecipitation mechanism.

(v) The solid grains are, at the conclusion of rearrangement, all of the same size, and randomly packed as an assembly of equisized spheres. We assume that there is no coarsening of the solid phase, despite the fact that coarsening is necessarily present to some extent in systems densifying by the solutionreprecipitation mechanism (we subsequently relax this assumption). The assumed solid powder geometry is realistic from an engineering standpoint [14], but differs from bimodal powder blends explored by some authors $[8,9,15,16]$.

The assumption that, at the conclusion of rearrangement, the solid particles are initially packed roughly as dense random spheres stands to reason since, when the liquidforming powder melts, capillary forces pull the refractory solid spheres together until they reach maximum packing, as noted by Kingery [3]. This assumption is in agreement with expcrimental data in the $\mathrm{Fe}-\mathrm{Cu}$ and MgO-Kaolin systems [6,7], and is also supported, as noted by German, by the rationalization that, above the volume fraction solid corresponding to random close packing, by analogy with rheocast structures, the apparent viscosity of the semi-solid slurry must increase dramatically, thus slowing further rearrangement significantly [17].

(vi) The compact densifies in vacuum. The influence of a finite gas pressure in pores is thus not addressed here. 
(vii) Although we take into account the influence of externally applied pressure during densification, we assume that this external pressure does not cause solid grain deformation by mechanisms other than solution-reprecipitation, such as creep and plastic deformation (such additional solid phase deformation was observed in one study Ref. [18]).

(viii) We assume that densification is limited by the rate of diffusion of one clement of the solid phase through the liquid. If there is significant solubility of the liquid phase primary elements in the solid grains, the influence of such solubility and ensuing diffusion into the solid on the densification rate is assumed to be negligible. We base this assumption on the observation that, although the thermodynamic driving force for such homogenization is large in comparison with capillary forces, the rate of solidstate diffusion is generally low. Furthermore, a lack of compositional uniformity in the solid does not influence the liquid/solid surface energy, since interfacial compositions in both liquid and solid are very rapidly brought everywhere to local chemical equilibrium values [8].

When rearrangement has stopped, solid particles are thus assumed to be random close packed spheres, all of radius $r$, separated by liquid which contains isolated pores. These solid grains are pulled against one another by the liquid/vapour interface, aided by applied pressure (if any), such that the chemical activity of the solid in narrow regions of interparticle near-contact is raised. As a consequence, solid diffuses through the liquid to regions remote from interparticle contacts, resulting in flattening of these contacts and densification of the powder compact, by the well-known solution/reprecipitation mechanism presented by Kingery [3]. The rate of densification therefore depends on the rate of local neck flattening at solid particle proximity regions, and on the geometrical distribution of necks, grains, and pores in the densifying powder compact.

\section{KINETICS OF DENSIFICATION}

\subsection{Microstructural relations}

We use the formalism developed by Arzt et al. $[10,11]$ to model the geometrical evolution of the solid particles. These, save for the thin liquid layer at flattened particle contact necks are, by themselves, analogous to a dry powder compact densifying in the "compaction limit", to use the terminology of Arzt [10]: solid material removed at neck regions is uniformly redistributed along the remainder of the solid particle surface area.
As the compact densifies, the average total area $a$ of the flattened interparticle contact regions therefore equals, per particle [12]:

$$
a Z=4 \pi r^{2} \frac{V_{\mathrm{s}}\left(V_{\mathrm{s}}-V_{\mathrm{s} .0}\right)}{\left(1-V_{\mathrm{s} .0}\right)}
$$

where $V_{\mathrm{s}}$ is the volume fraction solid, $r$ the initial particle radius, $Z$ the average number of interparticle contact areas per particle, and $V_{s, 0}$ is the initial volumc fraction of solid, present at the end of rearrangement and the beginning of densification by solution/reprecipitation.

After initial rapid rearrangement of the particles, we can assume, provided the part dimensions are small, that (i) Darcian flow of the liquid across the sample is rapid on the time-scale of the sintering process, and (ii) that microstructural gradients caused by the gravitational field (e.g. $[19,20]$ ) are negligible. As a consequence, the liquid pressure is everywhere the same, and pores in the liquid are necessarily everywhere of the same size, as depicted in Fig. 1(a). Calling $r_{\mathrm{p}}$ the (uniform) pore radius in the solid/liquid compact, the pressure in the liquid equals:

$$
P_{1}=-\frac{2 \sigma_{\mathrm{lv}}}{r_{\mathrm{p}}}
$$

It is known from studies of the deformation of wet porous media $[21,22]$ that the response of a solidliquid mixture to an external applied pressure $P_{\text {ap }}$ is, at volume fractions such that solid particles are surrounded by liquid, roughly the same as that of the dry solid phase subjected to an "effective" stress or pressure $P_{\mathrm{e}}$ (in the terminology used for the mechanics of porous media), which equals the applied pressure minus that in the liquid:

$$
P_{\mathrm{e}}=P_{\text {ap }}-P_{1} .
$$

Therefore, using equation (6) of Ref. [11], if an external pressure $P_{\text {ap }}$ is applied on the liquid/solid mixture, the average force $f$ at interparticle necks is about the same as that which would be obtained in a dry powder compact made of the same solid particles and subjected to an applied pressure $P=P_{\mathrm{e}}$. In the liquid/solid powder compact, $f$ is therefore given by:

$$
f=\frac{4 \pi r^{2}}{Z V_{\mathrm{s}}}\left(P_{\text {ap }}+\frac{2 \sigma_{\mathrm{lv}}}{r_{\mathrm{p}}}\right) .
$$

When $f$ is positive, each particle changes shape: it becomes flat at contact neck areas, and remains round elsewhere. We assume that the curvature of the particles at non-flattened liquid/solid interface areas is essentially constant across the compact. We base this assumption on the argument that transport of the solid material through wider liquid pools is much more rapid than transport through the narrow trough of liquid separating two nearly contacting particles. Equalization of curvature along curved solid/liquid interface regions is therefore rapid on the time scale of sintering. 
The particles do not change their volume during densification (if we ignore the very small variations in the quantity of solid dissolved in the liquid); hence their average surface curvature does not depart significantly from $2 r^{-1}$, the original curvature of the unsintered spherical particles. Consequently, we can write that, within the approximations of this model, the curvature $C$ along the solid/liquid interface in non-flattened regions is roughly given by:

$$
4 \pi r^{2}\left(\frac{2}{r}\right)=\left(4 \pi r^{2}-a Z\right) C
$$

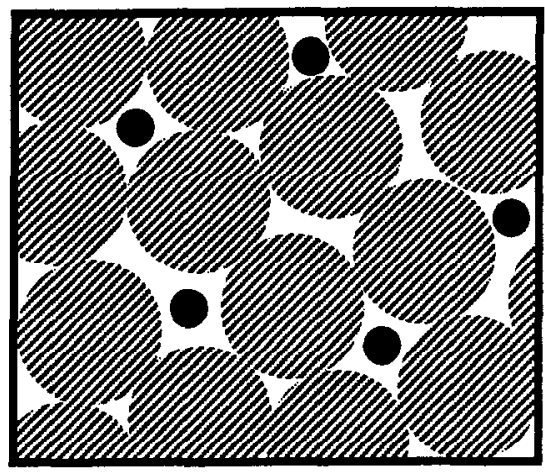

(a)

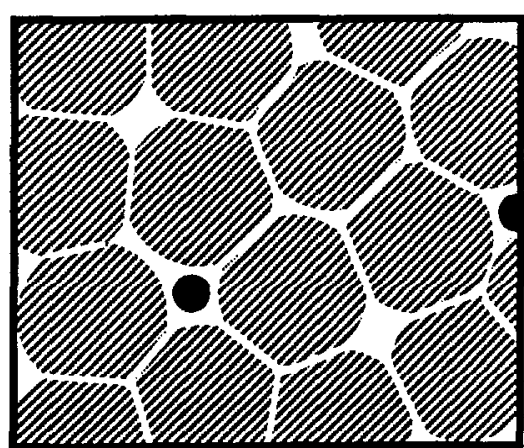

(b)

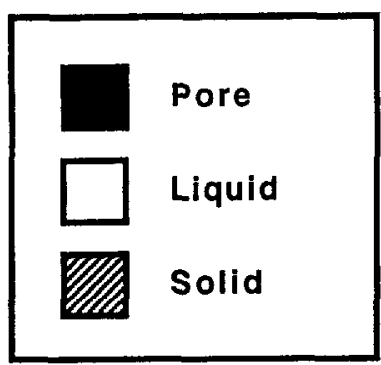

Fig. 1. Schematic description of the microstructural evolution during sintering by solution-reprecipitation: (a) initial structure at the end of rearrangement; (b) after partial sintering.
Hence:

$$
C=\frac{2}{r\left(1-\frac{a Z}{4 \pi r^{2}}\right)}
$$

$C$ is larger than $2 R^{-1}$, equalling this value before initiation of densification, and tending to a very large value if densification proceeds to a point where nearly all of the solid/liquid interface area is located along flattened contact areas. The limiting behaviour of equation (8) is, thus, correct.

The driving force for densification in the solution/ reprecipitation mechanism is the difference in frec energy of the solid between locations along the curved solid/liquid interface on the one hand, and that at flat interparticle contact necks on the other. This, in turn, is proportional to the difference in local pressure in the solid at each of these locations:

$$
\Delta P=f / a-\sigma_{\mathrm{sl}} C
$$

Inserting equations (3), (6) and (8), equation (9) becomes:

$$
\begin{aligned}
\Delta P=\frac{\left(1-V_{\mathrm{s}, 0}\right)}{V_{\mathrm{s}}^{2}\left(V_{\mathrm{s}}-V_{\mathrm{s}, 0}\right)}\left(P_{\mathrm{ap}}+\frac{2 \sigma_{\mathrm{lv}}}{r_{\mathrm{p}}}\right) & \\
& -\frac{2 \sigma_{\mathrm{s}}\left(1-V_{\mathrm{s}, 0}\right)}{r\left(\left(1-V_{\mathrm{s}, 0}\right)-V_{\mathrm{s}}\left(V_{\mathrm{s}} V_{\mathrm{s}, 0}\right)\right)}
\end{aligned}
$$

We note that if $P_{\mathrm{ap}}=0$ and $r_{\mathrm{p}}$ tends to infinity, $V_{\mathrm{s}}$ must approach $V_{\mathrm{s}, 0}$ : this stands to reason, since this case is that of solid particles fully immersed in a large quantity of liquid with no applied stress. The last term on the right-hand side of equation (10) rcpresents the increase in free energy corresponding to the departure from sphericity imposed on solid particles for densification to occur. This term thus represents the "sphering force" discussed in the literature [2]: its presence reduces the driving force for densification, as expected.

Equation (10) gives the driving force for liquid phase densification by solution/reprecipitation in a non-coarsening system, with inclusion of the influence of externally applied stress and of particle excess surface energy. Coupled with a local description of solid phase removal from neck areas, it provides a prediction of the rate of densification.

\subsection{Kinetics of densification by solution-reprecipitation}

The difference in pressure $\Delta P$ between interparticle contact regions and solid/liquid pool interfaces causes a difference in concentration in the solid between these two regions. This difference in concentration can be evaluated simply if we assume, as is generally done, that the solid phase is pure, and that its concentration in the liquid phase is relatively small. If we then consider the case where interfacial 
kinetics are rapid, with the implication that the rate of densification is limited by diffusion of the solid through the liquid, local concentration differences in the liquid along the solid are given by:

$$
R T \ln \left(C_{\text {neck }} / C_{\text {pools }}\right)=V_{0} \Delta P,
$$

where $R$ is the gas constant, $T$ is temperature, $V_{0}$ is the partial molar volume of the solid in the liquid, and $C_{\text {neck }}$ and $C_{\text {pool }}$ are concentrations of the solid phase diffusing element in the liquid at the neck and pool regions, respectively. Since $C_{\text {neck }}$ and $C_{\text {pool }}$ generally remain close to $C_{n}$, where $C_{0}$ is the equilibrium solid diffusing element concentration in the liquid at equilibrium with a flat interface (given by the liquidus composition at temperature $T$ ), we can simplify equation (11) as:

$$
\Delta C=C_{0} V_{0} \Delta P(R T)^{-1}
$$

where $\Delta C=C_{\text {neek }}-C_{\text {pool }}$. The rate of diffusion of the solid from the necks to the remaining liquid/solid interface regions is limited by the rate of diffusion through the narrow liquid channels at neck regions. Therefore, the volumetric rate of removal of the solid phase by diffusion through the liquid trough separating particles at neck regions is given by the same governing equations as for solid-state densification by grain boundary diffusion. save for the fact that the average pressure at particle contact areas in the solid powder compact $\left(P_{\text {eff }}\right.$ in the notation of Refs [10, 12]) is to be replaced with $\Delta P$ of equation (10).

Using the approximate expressions given in equations (8) and (19) of Ref. [12], which are very close to the full solution for grain-boundary diffusion limited solid state densification, but algebraically far simpler, we deduce:

$$
\frac{\mathrm{d} V_{\mathrm{s}}}{\mathrm{d} t}=\frac{43 \delta D C_{0} V_{\mathrm{i}}}{r^{3} R T} \frac{\left(1-V_{\mathrm{s}, 0}\right) V_{\mathrm{s}}^{2}}{\left(V_{\mathrm{s}}-V_{\mathrm{s}, 0}\right)} \Delta P
$$

which becomes, after insertion of equation (10):

$$
\begin{aligned}
& \frac{\mathrm{d} V_{\mathrm{s}}}{\mathrm{d} t}=\frac{43 \delta D C_{0} V_{0}\left(1-V_{\mathrm{s}, 0}\right)^{2}}{r^{3} R T}\left[\frac{P_{\mathrm{ap}}+\frac{2 \sigma_{\mid \mathrm{v}}}{r_{\mathrm{p}}}}{\left(V_{\mathrm{s}}-V_{\mathrm{s} .0}\right)^{2}}\right. \\
& \left.-\frac{2 \sigma_{\mathrm{s} 1}}{r} \frac{V_{\mathrm{s}}^{2}}{\left(V_{\mathrm{s}}-V_{\mathrm{s}, 0}\right)\left(1-V_{\mathrm{s}, 0}-V_{\mathrm{s}}\left(V_{\mathrm{s}}-V_{\mathrm{s}, 0}\right)\right)}\right] .
\end{aligned}
$$

An important microstructural parameter that remains to be known for prediction of the kinetics of liquid phase densification is the pore radius $r_{\mathrm{p}}$ within the densifying compact.

The smallest expected pore size would be derived by assuming that, throughout densification, the number of pores remains constant with approximately one pore per interparticle interstice. The corresponding value for $r_{p}$ can then be deduced by analogy with a highly dense solid particle compact, by assimilating the solid and liquid phases combined to a solid powder in the final stages of densification. This would be unrealistic, however, because pores can coarsen very rapidly in a semi-solid powder compact. If one pore is slightly smaller than its neighbours, it will not equalize its radius with that of other pores, but will instead shrink to disappear at a rate limited only by Darcian liquid flow, which is rapid across small distances such as a few times $r$. Therefore, variations in initial pore size, which must always be present in the powder compact after melting of the liquid and initial rearrangement, will cause the suppression of a large number of pores early in the densification process.

It thus seems more logical to assume that pores rapidly become much larger and far fewer than would be expected on the assumption that each area left between solid particles contains one pore. This is indeed observed in practice (see for example fig. 5-2, p. 103 of Ref. [2]).

We therefore assume that pores in the liquid coarsen rapidly during the initial rearrangement stage, to reach a stable radius $r_{\mathrm{p}}$ equal to the larger spaces present between the solid particles. Unless melting phase particles of radius significantly in excess of the solid particle radius $r$ are used (which could result in the presence of large empty spaces between bridging solid particles, and would, hence, be unwise from a practical standpoint), $r_{\mathrm{p}}$ is somewhat smaller than, but not far from, the radius of solid particles $r$. We therefore assume that $r_{\mathrm{p}}=r / 2$. This approximate value agrees for example with the micrograph on p. 103 of Ref. [2], is the same as that adopted by Kingery [3], agrees with the assessment by Eremenko et al. p. 55 that $r_{\mathrm{p}}$ is close to $r$ [1], and is well within the uncertainty inherent in other assumptions or approximations of the present analysis.

The sintering rate equation then becomes:

$$
\begin{aligned}
& \frac{\mathrm{d} V_{\mathrm{s}}}{\mathrm{d} t}=\frac{43 \delta D C_{0} V_{0}\left(1-V_{\mathrm{s}, 0}\right)^{2}}{r^{3} R T}\left[\frac{P_{\mathrm{ap}}+\frac{4 \sigma_{\mathrm{lv}}}{r}}{\left(V_{\mathrm{s}}-V_{\mathrm{s}, 0}\right)^{2}}\right. \\
& \left.-\frac{2 \sigma_{\mathrm{s} 1}}{r} \frac{V_{\mathrm{s}}^{2}}{\left(V_{\mathrm{s}}-V_{\mathrm{s}, 0}\right)\left(1-V_{\mathrm{s}, 0}-V_{\mathrm{s}}\left(V_{\mathrm{s}}-V_{\mathrm{s}, 0}\right)\right)}\right]
\end{aligned}
$$

If we focus for simplicity on densification by sintering, i.e. if we assume that $P_{\text {ap }}=0$, equation (15) can be rewritten in terms of dimensionless numbers as:

$$
\begin{aligned}
\frac{\mathrm{d} V_{\mathrm{s}}}{\mathrm{d}(K t)} & =86\left(1-V_{\mathrm{s}, 0}\right)^{2}\left[\frac{2}{\left(V_{\mathrm{s}}-V_{\mathrm{s}, 0}\right)^{2}}\right. \\
& \left.-\frac{s V_{\mathrm{s}}^{2}}{\left(V_{\mathrm{s}}-V_{\mathrm{s}, 0}\right)\left(1-V_{\mathrm{s}, 0}-V_{\mathrm{s}}\left(V_{\mathrm{s}}-V_{\mathrm{s}, 0}\right)\right)}\right]
\end{aligned}
$$




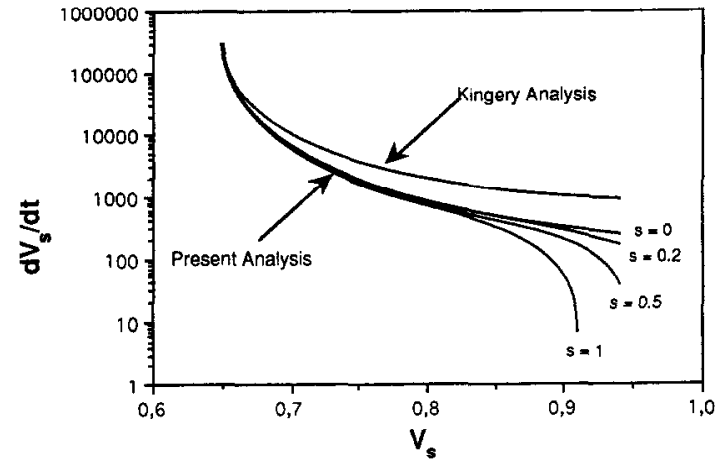

Fig. 2. Influence of the "sphering force" on the rate of densification by liquid phase sintering as given by equation (16), and comparison with the model of Kingery.

with

$$
K=\frac{\delta D C_{0} V_{0} \sigma_{\mathrm{lv}}}{r^{4} R T}
$$

and

$$
s=\frac{\sigma_{\mathrm{sl}}}{\sigma_{\mathrm{lv}}}
$$

Parameter $s$ is generally smaller than unity, because the surface energies of liquids significantly exceed liquid/solid interfacial energies, by about an order of magnitude. Although $s$ will clearly vary from system to system, it is generally expected that $s \leqslant 0.2$.

A plot of $\mathrm{d} V_{\mathrm{s}} / \mathrm{d}(K t)$ for sintering with no applied pressure is given in Fig. 2. It is seen that with $s \leqslant 0.2$, the second term between brackets in equation (16), which expresses the influence of stored capillary energy due to shape accommodation of the solid particles, exerts only a small influence on densification kinetics. Since applied pressure increases the driving force for densification, this conclusion is a fortiori valid for pressure-assisted densification by solutionreprecipitation.

If we then neglect the second term between brackets in equation (16), this equation can be simplified and integrated, to yield a reasonably accurate and algebraically very simple prediction of densification kinetics in liquid phase sintering by solution reprecipitation:

$$
\left(V_{\mathrm{s}}-V_{\mathrm{s}, 0}\right)=\left[516\left(1-V_{\mathrm{s}, 0}\right)^{2} K t\right]^{1 / 3} .
$$

If we consider a compact containing a volume $V$ of the solid phase, the total compact volume is, at every instant, $V V_{\mathrm{s}}^{-1}$. Calling $L$ a given characteristic length (e.g. the diameter or the height) of the compact at time $t$, at every instant $L$ is therefore proportional to $V_{s}^{-1 / 3}$. The total relative sample shrinkage is, thus, related to $V_{\mathrm{s}}$ and $V_{\mathrm{s}, 0}$ by:

$$
\frac{\Delta L}{L_{0}}=1-\left(\frac{V_{\mathrm{s}, 0}}{V_{\mathrm{s}}}\right)^{1 / 3}
$$

where $L_{0}$ is the initial value of $L$. Insertion of equation (19) in equation (20) thus yields the rate of densification as measured by the apparent relative linear shrinkage.

\section{DISCUSSION}

\subsection{Comparison with Kingery's model and with experimental data}

Kingery's equation, derived under the implicit assumption that solid particles in the compact are packed in a regular lattice, and also assuming that $r_{\mathrm{p}}=r / 2$, yields (equation (19) of Ref. [3] with $\left.k_{1}=1 / 2\right)$ :

$$
\frac{\Delta L}{L_{0}}=(12 K t)^{1 / 3}
$$

A comparison of the two models is given in Fig. 3 in terms of $V_{\mathrm{s}}$ and, in Fig. 4 , in terms of the linear shrinkage $\Delta L / L_{0}$, this last plot reproducing the now classical format used by Kingery. The two expressions are relatively close, both yielding nearly straight lines on a plot of $\log \left(\Delta L / L_{0}\right)$ versus $\log (t)$, see Fig. 4. The third-root of time behaviour found by Kingery is reproduced at small $K t$ in the present model, as can be deduced analytically by inserting equation (20) into equation (19) and taking the limit at small $t$.

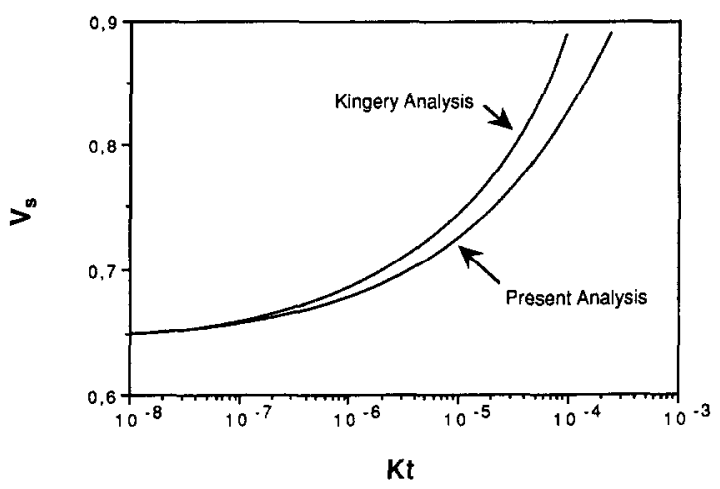

Fig. 3. Rate of densification by liquid phase sintering in terms of the volume fraction solid phase $V_{s}$, as predicted by the present model and by the model of Kingery.

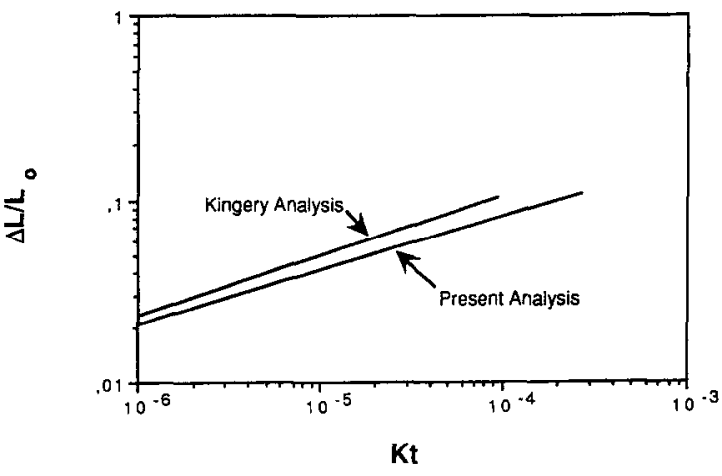

Fig. 4. Rate of densification by liquid phase sintering in terms of the linear shrinkage rate, as predicted by the present model and by the model of Kingery. 
At longer time and greater $V_{\mathrm{s}}$, corresponding to $K t$ greater than about $10^{-5}$, the predicted rate of sintering decreases somewhat below $1 / 3$, but remains above 0.25 . This predicted slight downwards deviation from the power-law of Kingery at longer sintering times is due to the creation of new sphere-to-sphere contacts, which in liquid phase sintering slow densification because no added driving force is associated with their creation (contrary to what is obtained in solid state sintering).

The dependence of the rate of sintering on system parameters and on particle radius $r$ is the same in the current analysis as in that of Kingery, since in both analyses this dependence is embodied in the same dimensionless quantity $K t$. In particular, both analyses predict that the sintering rate is proportional to $r^{-4,3}$ in pressureless sintering. The effect of applied pressure $P_{\text {ap }}$, given in equation (15) by the present derivation, is also in agreement with that found by Kingery via consideration of two contacting particles only (equation (3) of Ref. [5]).

The overall agreement between the present analysis and the predictions from Kingery's model is very satisfactory, given the ample amount of experimental data showing that $\log \left(\Delta L / L_{0}\right)$ is, indeed, proportional to $\log (t)$, with a slope equal to, or slightly below, one-third during sintering by solution-reprecipitation after rearrangement $[1,2,6,7], \dagger$ and that observed sintering rates do indeed vary proportionally with $r^{-4 / 3}[6]$.

Full quantitative comparison of predicted and measured densification rates is difficult given the uncertainty in governing parameters, especially $\delta D$. Using realistic values of these parameters, Kingery found rather good agreement between equation (21) and sintering rates measured in the iron copper system, his prediction being in excess of the experiment by a factor nearly two. The present analysis improves the agreement by lowering the predicted rates (Figs 3 and 4) by a factor indeed near two; however, as noted by Kingery, such excellent agreement is probably fortuitous given the uncertainty in the evaluation of $K$. We thus conclude that agreement between theory and published data is as good as can be hoped for, given the uncertainty on the effective diffusion constant $\delta D$.

\subsection{Influence of coarsening}

Liquid/solid materials systems in which densification can be driven by the solution-reprecipitation process are ones in which the solid phase coarsens as well, because the two phenomena are caused by the same physical process of solid phase dissolution and redeposition. When transport of the solid through the

†We note that, to confirm this law rigorously, experimental data should have been plotted with $t=0$ and $L_{0}$ defined after completion of the rearrangement rather than at the initiation of sintering; however, this should not invalidate this rather general observation. liquid is limited by diffusion, as was assumed in the previous section, the dependence of particle radius $r$ on time $t$ follows the well known relation:

$$
r^{3}=r_{0}^{3}+k t
$$

where $r_{0}$ is the initial solid particle radius, and $k$ is a constant, which varies somewhat with the volume fraction of solid phase present (and, thus, during sintering); however, with adequate precision, $k$ is roughly given by $[2,23,24]$ :

$$
k \approx \frac{2 D C_{0} V_{0} \sigma_{\mathrm{ls}}}{R T}
$$

for liquid-solid compacts of volume fraction solid somewhat above $50 \%$ (i.e. about four times the value predicted by the classical LSW analysis for very low $V_{\mathrm{s}}$ ).

In the presence of coarsening, the present analysis can be extended if it is assumed that, while particles coarsen, their distribution remains that of randomly close-packed spheres. This assumption is necessarily an approximation, since it requires slight rearrangement of particle centres as densification progresses, and hence that there is some slight change in neck region shapes with time. Solid particle radii also cannot, in the presence of coarsening, assume a single value, but are distributed within a relatively narrow range of sizes about the average radius value, $r$ : we ignore the influence of this slight spread in particle radius on particle centre distribution.

Proceeding as above, but with a time-dependent particle radius $r$ and maintaining the assumption that $r_{\mathrm{p}} \approx r / 2$, equation (14) is replaced by:

$$
\begin{aligned}
\frac{\mathrm{d} V_{\mathrm{s}}}{\mathrm{d} t} & =\frac{43 \delta D C_{0} V_{0}\left(1-V_{\mathrm{s}, 0}\right)^{2}}{\left(r_{0}^{3}+k t\right)^{4 / 3} R T}\left[\frac{\left(r_{0}^{3}+k t\right)^{1 / 3} P_{\mathrm{ap}}+4 \sigma_{\mathrm{lv}}}{\left(V_{\mathrm{s}}-V_{\mathrm{s}, 0}\right)^{2}}\right. \\
& \left.-\frac{2 \sigma_{\mathrm{sl}} V_{\mathrm{s}}^{2}}{\left(V_{\mathrm{s}}-V_{\mathrm{s}, 0}\right)\left(1-V_{\mathrm{s}, 0}-V_{\mathrm{s}}\left(V_{\mathrm{s}}-V_{\mathrm{s}, 0}\right)\right)}\right] \cdot
\end{aligned}
$$

If we focus again on densification by sintering only, and again neglect the second term between brackets in equation (24), this equation can be simplified and integrated to become:

$$
\begin{aligned}
\left(V_{\mathrm{s}}-V_{\mathrm{s}, 0}\right)= & {\left[\begin{array}{rl}
1548\left(1-V_{\mathrm{s}, 0}\right)^{2} \frac{K r_{0}^{3}}{k} \\
\left.\times\left(1-\frac{1}{\left(1+\frac{k}{K r_{0}^{3}} K t\right)^{1 / 3}}\right)\right]^{1 / 3}
\end{array}\right.} \\
& \times\left(1-\frac{1}{(1)}\right.
\end{aligned}
$$

or, after insertion of equation (23):

$$
\left(V_{\mathrm{s}}-V_{\mathrm{s}, 0}\right)=\left[\frac{1548\left(1-V_{\mathrm{s}, 0}\right)^{2}}{w}\left(1-\frac{1}{(1+w K t)^{1 / 3}}\right)\right]^{1,3}
$$




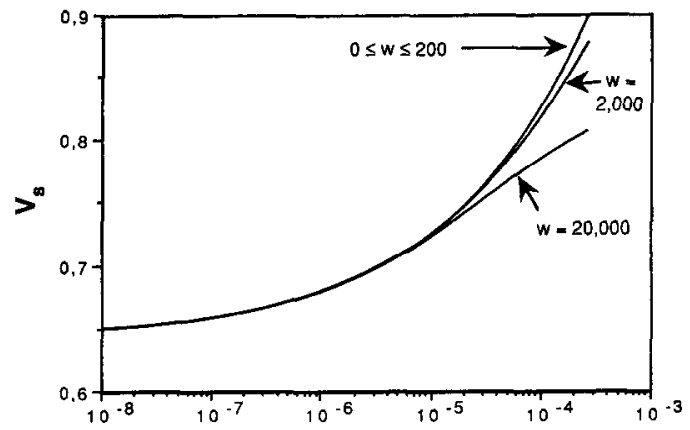

Kt

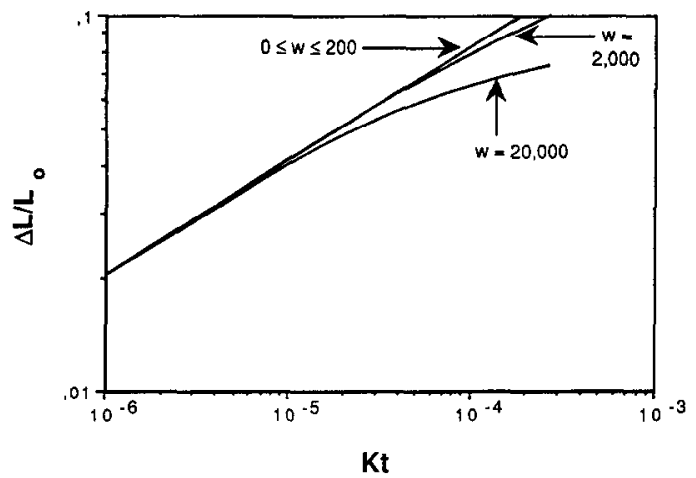

Fig. 5. Predicted rate of densification in the presence of coarsening for various values of parameter $w$ : (a) in terms of the volume fraction solid phase $V_{s}$; (b) in terms of the linear shrinkage rate.

with

$$
w=\frac{2 r_{0} s}{\delta} .
$$

The resulting densification curves are shown in Fig. 5 for various values of $w$ : it is seen that for coarsening to influence significantly the rate of densification, $w$ must exceed 2000.

As stated above, $s$ is approximately of the order of 0.2. For coarsening to influence the rate of densification $r_{0}$ must, therefore, exceed $\delta$ by at least a factor of 5000. If, following Kingery [6], we estimate $\delta$ as being of the order of a nanometre, $r_{n}$ must then roughly exceed $5 \mu \mathrm{m}$ for coarsening to influence significantly the kinetics of sintering.

When this is the case, coarsening reduces the rate of densification compared with that which would be expected based on the initial particle size (but not the final solid particle size found in the final sintered microstructure). It is interesting to note the a priori somewhat counter-intuitive nature of the conclusion that coarsening becomes important with initially coarse solid particles. This arises because the time for sintering is proportional to the inverse of the fourth power of particle radius (equation (16)), so that the acceleration in sintering kinetics caused by small $r_{0}$ overrides its influence on coarsening. From a practical standpoint, therefore, the advantage inherent in the use of fine particles to speed sintering is amplified by the possible influence of coarsening.

\subsection{Influence of packing configuration}

The influence of the initial packing density is apparent in the term $V_{\mathrm{s}, 0}$, provided it is assumed that when $V_{\mathrm{s}, 0}$ deviates from 0.64 , variations in packing coordination and average neck surface are still appropriately described with the simplified relationships derived by Arzt $e t$ al , and used here. It has been observed [7] that inhomogeneous initial packing of the powders exerts a strong influence on densification kinetics: more homogeneously packed powders densify faster. This can be rationalized within the framework of the present analysis by noting that inhomogeneous initial powder packing may (i) decrease the volume fraction solid at the end of rearrangement, $V_{\mathrm{s}, 0}$; and (ii) create a few large spaces between solid particles, leaving sites for larger pores to be present, in turn causing $r_{\mathrm{p}}$ to increase. Both effects decrease the densification rate predicted by equations (14) and (24). That increased powder homogeneity increases $V_{s, u}$ has indeed been observed experimentally [25].

The amount of liquid present does not affect the rate of densification by solution-reprecipitation according to the present analysis (although it strongly influences the initial rearrangement stage, and determines when solution-reprecipitation has fully densified the powder compact). This is also in agreement with experimental observations [2, p. 107], [7] that the amount of liquid present does not influence the rate of densification significantly.

\subsection{Final microstructure}

As densification proceeds, the total volume of pores present in the powder compact decreases. With a relatively constant pore size, this implies that during densification, the pore number decreases, pores gradually disappearing as liquid can fill more space between densifying solid phase particles. Clearly, in this process, surviving pores will be the largest ones present, and $r_{\mathrm{p}}$ is therefore expected to increase somewhat with time. Significant pore coarsening of this nature would cause further deviations in the densification rate downwards of Kingery's $t^{1 / 3}$ law for linear compact shrinkage. Experiment shows no such gradual transition; instead, densification stops relatively abruptly once the $t^{1 / 3}$ law ceases to be obeyed. This indicates that pores remain roughly constant in size (as assumed here) until nearly all pores that drive densification disappear, due to filling by the liquid.

At the end of densification, barring any gross microstructural defects, the density of the compact is near one. Since the ratio of liquid to solid volumes in the compact remains constant at all times, we have:

$$
\frac{V_{1}}{V_{\mathrm{s}}}=\frac{V_{1,0}}{V_{\mathrm{s}, 0}} \equiv N,
$$

where $V_{1}$ is the volume fraction liquid in the compact, $V_{1,0}$ is the initial volume fraction liquid in the 
compact. In a dense compact, therefore, $V_{\mathrm{s}}$ is given by:

$$
V_{\mathrm{s}}=\frac{1}{1+N}
$$

When densification stops, there are few or no pores left in the powder compact, but the solid particles are deformed. In the resulting metastable structure, the liquid remains under hydrostatic tension; therefore, $r_{\mathrm{p}}$ docs not decay to zero when densification ceases, as pointed out previously by several authors [2, p. 104], [26]. If we consider a fully densified powder compact, having densified for simplicity by sintering, i.e. with $\Delta P_{\text {ap }}=0$, the microstructure ceases to evolve when $\Delta P=0$, i.e. when:

$r_{\mathrm{p}}=\frac{r}{S}(1+N)^{2} \frac{1-V_{\mathrm{s} .0}-(1+N)^{-1}\left((1+N)^{-1}-V_{\mathrm{s} .0}\right)}{\left((1+N)^{-1}-V_{\mathrm{s}, 0}\right)}$.

Equation (30) provides, for a given dense compact, the radius of curvature of liquid menisci at the free surface of the compact (if we assume that these are more cylindrical than spherical, their radius of curvature is roughly equal to $r_{p} / 2$ ).

If there are any residual pores present in the compact, their overall radius must excecd $r_{\mathrm{p}}$ since for any pore of radius less than $r_{p}$, the local pressure in the liquid is necessarily below the average (negative) liquid pressure, so that the liquid is pulled inwards to fill the pore. Liquid-filled regions devoid of solid particles are a feature of liquid phase sintered structures that have, indeed, been observed experimentally [2, p. 120].

The equilibrium $r_{\mathrm{p}}$ value predicted by equation (28) is proportional to $r$; this is in agreement with previous analyses of the final stable pore size in compacts densified by solution-reprecipitation $[18,26]$ (these earlier analyses assumed spherical solid particles packed along a regular close-packed lattice). This conclusion also agrees with experimental data which, furthermore, indicate that as the liquid content (and hence $N$ ) increases, the smallest stable pore size increases [18].

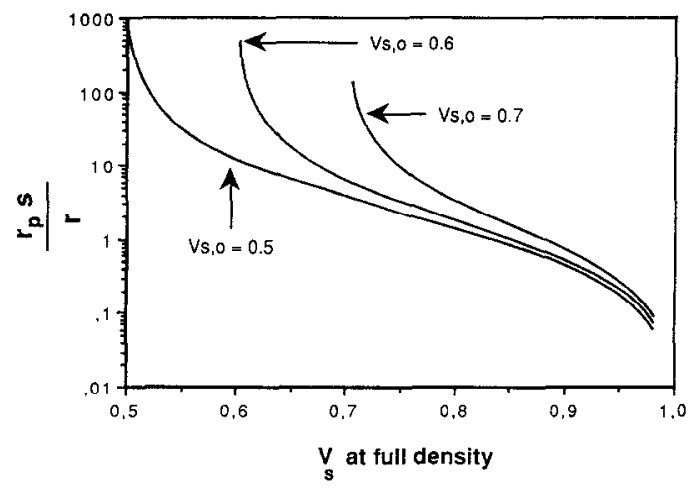

Fig. 6. Predicted values of $\left(r_{p} s / r\right)$ as a function of the liquid to solid phase volume ratio $N$.
A plot of $\left(r_{\mathrm{p}} s / r\right)$ versus $V_{\mathrm{s}}$ at full (or approximately full) density is given in Fig. 6 for various values of $V_{\mathrm{s}, 0}$. It is seen that as the amount of liquid relative to solid increases, i.e. that as $N$ increases, $r_{\mathrm{p}}$ indeed increases. This is because the more liquid is present in the compact, the less solid particles are pressed together when full density is attained. This, in turn, implies that the negative pressure required in the liquid to hold the particles pressed together (i.e. the "sphering pressure" in the terminology of Park et al. [26]), decreases as the amount of liquid present in the dense compact decreases; hence $r_{p}$, and the diameter of the largest unfilled pore in the final structure, increases as $N$ increases. It is also seen that for $V_{\mathrm{s}}$ at full density above about $0.8,\left(r_{\mathrm{p}} s / r\right)$ exceeds one. Knowing, as stated above, that $\sigma_{\mathrm{sl}}$ is generally smaller than $\sigma_{\mathrm{sv}}$ by up to one order of magnitude, $r_{\mathrm{p}}$ exceeds $r$ by about one order of magnitude in the fully densified microstructure. The calculation thus predicts that the smallest pores present in the compact after full densification by solution/reprecipitation will be significantly larger than the average particle diameter $r$ : this conclusion is in agreement with microstructural observations (e.g. [2, pp. 128-129], [7, 15]).

We finally note that the value given for $r_{\mathrm{p}}$ by equation (30), which in turn gives via equation (4) the liquid pressure in a fully densified structure, provides a quantitative prediction of the driving force for macroscopic liquid migration in layered or graded liquid phase sintered structures [27]. The rate of such migration, as well as microstructural gradients caused by gravity in liquid-phase sintered microstructures, can also be predicted on the basis of the present analysis.

\section{CONCLUSION}

Equations describing the microstructure and the rate of densification in liquid phase sintering by solution-reprecipitation are given for systems in which capillary forces are isotropic, and favour wetting of the solid by the liquid. $\Lambda$ s such, the analysis presented here represents an extension of Kingery's analysis, which relieves its simplified description of a powder compact in terms of two particles only.

Compared with Kingery's analysis, rates of densification predicted by the present analysis are lower by a factor below two, maintain approximately the prediction of a one-third power-law dependence of sintering rate on time in the absence of coarsening, and feature the same functional relationship with other parameters such as particle size. Agreement between theory and experiment is excellent in terms of parametric dependencies, and as good as can be hoped for in terms of absolute densification rates, given uncertainty in relevant parameters.

The analysis is extended to account for the influence of coarsening on densification kinetics. It is shown that the influence of coarsening on the rate of 
liquid phase sintering is noticeable when the width of the liquid layer separating solid particles along their contact regions falls below about one-thousandth of the initial solid particle radius. The influence of coarsening is, hence, pronounced with large solid particles.

In a fully densified compact, deviations in the shape of the solid particles from a sphere cause a build-up of negative pressure in the liquid. This pressure is predicted to provide an expression for the smallest stable pore radius in the densified structure.

Acknowledgements-This work was performed by the author while on sabbatical leave at the Centre des Matériaux Pierre-Marie Fourt of the Ecole Nationale Supérieure des Mines de Paris in France, under sponsorship from the French Ministry of Education and Research, in the form of a Bourse de Haut Niveau awarded to the author.

\section{REFERENCES}

1. V. N. Eremenko, Y. V. Naidich and I. A. Lavrinenko, Liquid Phase Sintering, Consultants Bureau, New York (1970).

2. R. M. German, Liquid Phase Sintering. Plenum Press, New York (1985).

3. W. D. Kingery, J. Appl. Phys. 30, 301 (1959).

4. W. D. Kingery and M. Berg, J. Appl. Phys. 26, 1205 (1995).

5. W. D. Kingery, J. M. Woulbroun and F. R. Charvat, J. Am. Cer. Soc. 46, 391 (1963).

6. W. D. Kingery and M. D. Narasimhan, J. Appl. Phys. 30, 307 (1959).

7. W. D. Kingery, E. Niki and M. D. Narasimham, J. Am. Cer. Soc. 44, 29 (1961).

8. W. J. Huppmann, Z. Metallk. 70, 792 (1979).

9. W. A. Kaysser and G. Petzow, Powder Metall. 28, 145 (1985).
10. E. Arzt, Acta Metall. 30, 1883 (1982).

11. E. Arzt, M. F. Ashby and K. E. Easterling, Metall. Trans. 14A, 211 (1983).

12. A. S. Helle, K. E. Easterling and M. F. Ashby, Acta Metall. 33, 2163 (1985).

13. M. F. Ashby, Powder Metallurgy: An Overview (edited by I. Jenkins and J. V. Wood), pp. 144-166. The Institute of Metals (1991).

14. R. M. German, Metall. Trans. 26A, 279 (1995).

15. D. N. Yoon and W. J. Huppmann, Acta Metall. 27, 693 (1979).

16. S. Taruta, T. Takano, N. Takusagawa, K. Okada and N. Otsuda, J. Mater. Sci. 31, 573 (1996).

17. R. M. German, in Proc. Conf. $P / M$ into the 90 's, Wembley Conference Centre, London, UK (edited by The Institute of Metals, London, U.K.), pp. 172-184 (1990).

18. C. C. Ge and Y. L. Xia, in Proc. Conf. Int. Powder Metall. Conf-Modern Developments in Powder Metallurgy Vol. 15, Toronto, Canada (edited by E. N. Aqua and C. I. Whitman), pp. 455-476, Metal Powder Industries Federation American Powder Metallurgy Institute (1984).

19. C. Kipphut, A. Bose, S. Farooq and R. M. German, Metall. Trans. 19A, 1905 (1988).

20. Y. Liu, D. F. Heaney and R. M. German, Acta Metall. Mater. 43, 1587 (1995).

21. A. Nur and J. D. Byerlee, J. Geophys. Res. 76, 6414 (1971).

22. J. Bear, Dynamics of Fluids in Porous Media, American Elsevier, New York, p. 57. (Re-edited by Dover Pess, New York) (1972).

23. P. W. Voorhees and M. E. Glicksman, Metall. Trans. 15A, 1081 (1984).

24. A. Mortensen, Metall. Trans. 22A, 569 (1991).

25. W. J. Huppmann, H. Riegger, W. A. Kaysser, V. Smolej and S. Pejovnik, Z. Metallk. 70, 707 (1979).

26. H. H. Park, S. J. Cho and D. N. Yoon, Metall. Trans. 15A, 1075 (1984).

27. A. Mortensen and S. Suresh, Int. Mater. Rev. in print (1996). 